\title{
Análise bayesiana na estimação de correlações genéticas entre escores visuais e características reprodutivas de bovinos Nelore utilizando modelos linear-limiar
}

\author{
[Bayesian analysis in the estimation of genetic correlations between visual scores and reproductive \\ traits in Nelore cattle using linear-threshold models] \\ C.U. Faria ${ }^{1}$, C.U. Magnabosco ${ }^{2}$, L.G. Albuquerque ${ }^{3}$, A. de los Reyes ${ }^{1}$, L.A.F. Bezerra ${ }^{4}$, R.B. Lôbo ${ }^{4,5}$ \\ ${ }^{1}$ Universidade Federal de Goiás - UFG - Campus de Jataí \\ BR 364, Km 192, Caixa Postal 03 \\ 75800-000 - Jataí, GO \\ ${ }^{2}$ Embrapa Cerrados - Brasília, DF \\ ${ }^{3}$ Faculdade de Ciências Agrárias e Veterinárias - UNESP - Jaboticabal, SP \\ ${ }^{4}$ Faculdade de Medicina de Ribeirão Preto - USP - Ribeirão Preto, SP \\ ${ }^{5}$ Associação Nacional de Criadores e Pesquisadores - Ribeirão Preto, SP
}

\begin{abstract}
RESUMO
Estimaram-se as correlações genéticas entre os escores visuais e as características reprodutivas, utilizando a estatística bayesiana sob modelo animal linear-limiar, em bovinos da raça Nelore. Foram estudadas características categóricas morfológicas, avaliadas visualmente aos oito, 15 e 22 meses de idade; e características contínuas de perímetro escrotal padronizado aos 365 e 450 dias de idade, além da idade ao primeiro parto. As estimativas de correlações genéticas foram de sentido favorável à seleção, apresentando magnitudes moderadas, sugerindo que a seleção de animais para um biótipo desejável pode levar a animais com maior fertilidade e precocidade sexual. As estimativas de correlação genética para o perímetro escrotal padronizado aos 450 dias e a idade ao primeiro parto com as características morfológicas avaliadas aos 22 meses de idade foram maiores do que as obtidas entre as características de escores visuais avaliadas aos oito e 15 meses de idade. A utilização de escores visuais como critério de seleção trará progresso genético também para as características reprodutivas.
\end{abstract}

Palavras-chave: gado de corte, amostragem de Gibbs, características morfológicas, parâmetros genéticos

\begin{abstract}
The genetic correlations between visual scores and reproductive traits, using the bayesian statistic under the linear-threshold animal model, in bovines of the Nelore breed were estimated. Categorical morphological traits were also evaluated at eight, 15, and 22 month-old, concerning musculature, physical structure, conformation and sacrum, the escrotal circumferences measured at the ages of 365 and 450-day-old, and the age at first calving. The estimates of genetic correlations were moderates, suggesting that the selection of animals with better morphological conformation may lead to animals more precocious and with greater fertility. The estimates of genetic correlation concerning the escrotal circumference at 450 day-old and the age at first calving with the evaluated visual scores at 22 month-old were higher than the ones obtained between visual scores at eight and 15 month-old. The use of these visual scores as a criterion of selection may also bring genetic progress to the reproductive traits.
\end{abstract}

Keywords: beef cattle, Gibbs sampling, morphological traits, reproductive, traits

Recebido em 1 de julho de 2008

Aceito em 18 de maio de 2009

E-mail: carina@jatai.ufg.br 


\section{INTRODUÇÃO}

A produção de carne de forma eficiente é o principal objetivo da pecuária de corte. Estratégias seletivas que visem equilibrar o crescimento, o biótipo e a reprodução são desejáveis tanto no aspecto genético como no econômico.

Atualmente, a maioria dos estudos em precocidade sexual está relacionada ao perímetro escrotal e à idade ao primeiro parto. Dessa forma, a seleção para essas características poderia implicar ganhos genéticos para a eficiência reprodutiva. A eficiência reprodutiva é a característica econômica de maior importância em um rebanho (Pereira et al., 2001). No entanto, a precocidade de terminação talvez esteja correlacionada com a precocidade sexual.

Trabalhos que relacionam dimensões corporais com o desempenho reprodutivo em zebuínos são escassos na literatura, podendo ser citados os de Pita et al. (1998) e Semmelmann et al. (2001), em que foram estimadas somente as correlações fenotípicas entre essas características. Conhecer as estimativas de parâmetros genéticos é necessário para estimar as respostas diretas e correlacionadas à seleção, elaborar índices de seleção e predizer o valor genético dos animais.

O emprego de metodologias adequadas para obtenção de correlações genéticas entre as características categóricas morfológicas e as contínuas de reprodução, por meio de análises multicaracterísticas, é de grande interesse ao melhoramento genético animal e pode trazer maior contribuição para a pecuária de corte. Com base no conhecimento das consequências da seleção de uma característica em relação às outras, poder-se-á elaborar índices de seleção que identifiquem genótipos mais adequados aos distintos sistemas de produção praticados no Brasil.

$\mathrm{Na}$ avaliação genética de características morfológicas, em que os dados não apresentam uma distribuição normal, alguns cuidados devem ser considerados, uma vez que as metodologias de modelos lineares geralmente resultam em baixas estimativas de herdabilidade (Mwansa et al., 2002; Marcondes et al., 2005). Assim, recomenda-se o uso de modelos de limiar por apresentarem maior habilidade para detecção da variabilidade genética quando comparados aos modelos lineares (Luo et al., 2002).

O modelo de limiar oferece vantagens sobre o modelo linear nas análises para dados categóricos sob modelo animal (Ramirez Valverde et al., 2001). O modelo animal, por utilizar informações de todos os indivíduos e considerar as relações de parentesco existentes entre os animais em avaliação, promove maior acurácia nas estimativas do mérito genético. Além disso, possibilita a obtenção da estimativa da capacidade genética de transmissão para todo indivíduo avaliado, mesmo para animais jovens e, por conseguinte, sem progênie. De acordo com Mercadante et al. (2000), o modelo animal também é mais vantajoso para características de menor herdabilidade. A implementação de análise multicaracterística adiciona valores para essas características, especialmente quando são correlacionadas com características de maior herdabilidade (Meyer et al., 1991).

O objetivo deste estudo foi estimar as correlações genéticas entre as características morfológicas e de reprodução, utilizando a estatística bayesiana sob modelo animal linearlimiar, em bovinos da raça Nelore.

\section{MATERIAL E MÉTODOS}

As informações de escores visuais analisadas no presente estudo foram obtidas nos anos de 2000 a 2005, em bovinos da raça Nelore, provenientes de 13 fazendas participantes do Programa Nelore Brasil, da Associação Nacional de Criadores e Pesquisadores (ANCP), localizadas nos estados de Goiás, Mato Grosso e Paraná e no Distrito Federal. Foram estudadas as características categóricas morfológicas, avaliadas aos oito, 15 e 22 meses de idade, para musculosidade $\left(\mathrm{M}_{8}, \mathrm{M}_{15}\right.$, $\left.\mathrm{M}_{22}\right)$, estrutura física $\left(\mathrm{E}_{8}, \mathrm{E}_{15}, \mathrm{E}_{22}\right)$, conformação $\left(\mathrm{C}_{8}, \mathrm{C}_{15}, \mathrm{C}_{22}\right)$ e sacro $\left(\mathrm{SAC}_{8}, \mathrm{SAC}_{15}, \mathrm{SAC}_{22}\right)$; e as características contínuas de reprodução, perímetro escrotal padronizado aos 365 (PE365) e 450 (PE450) dias de idade, além da idade ao primeiro parto (IPP). As informações obtidas para as características de reprodução foram fornecidas pelo Programa Nelore Brasil.

Os animais foram avaliados visualmente pelo método denominado MERCOS, adaptado por Lôbo (1996), em que: (i) para musculosidade (M) avaliam-se a distribuição muscular no corpo 
do animal, e o desenvolvimento, volume e comprimento dos músculos; (ii) para estrutura física (E) avalia-se a sustentação do animal, ou seja, integridade e tamanho dos cascos e firmeza e largura dos ossos ligamentos ou articulações; iii) para conformação (C) avaliam-se o esqueleto, o comprimento do corpo, a amplitude torácica, o arqueamento e o comprimento das costelas, a largura de garupa e o comprimento, a relação tamanho dos membros e o comprimento das costelas. De acordo com Lôbo (1996), para cada variável morfológica podem-se obter escores de um a cinco pontos, sendo que a maior pontuação representa o grau mais favorável. $\mathrm{Na}$ aplicação do método de avaliação visual, os animais foram analisados individualmente pelo mesmo avaliador, para cada lote de manejo, o que caracterizou animais de mesmo sexo e nascidos na mesma época, e que receberam as mesmas condições de tratamento alimentar e sanitário.

Para atribuir escores para sacro (SAC), avaliouse o comprimento, por meio da distância entre os ossos íleo e ísquio, a largura, considerando a distância entre os íleos e entre os ísquios, e a inclinação do osso sacro. Como SAC é uma característica binária ou dicotômica, foram utilizados apenas dois escores. Assumiu-se valor um para animais com osso sacro indesejável e valor dois para osso sacro desejável. Na Tab. 1, é apresentada a distribuição dos escores em porcentagem para cada característica morfológica.

Tabela 1. Distribuição (\%) por escores para as características musculosidade (M), estrutura física (E), conformação (C) e sacro (SAC) avaliadas aos oito, 15 e 22 meses de idade em bovinos da raça Nelore

\begin{tabular}{lrrrrc}
\hline \multirow{2}{*}{ Característica } & \multicolumn{5}{c}{ Escore } \\
\cline { 2 - 6 } & $\mathrm{Um}$ & Dois $^{\mathrm{a}}$ & Três & Quatro & Cinco $^{\mathrm{b}}$ \\
\hline $\mathrm{M}_{8}$ & 5,5 & 28,8 & 50,4 & 14,3 & 1,0 \\
$\mathrm{E}_{8}$ & 3,4 & 24,5 & 45,8 & 24,9 & 1,4 \\
$\mathrm{C}_{8}$ & 5,5 & 35,1 & 45,5 & 13,6 & 0,3 \\
$\mathrm{SAC}_{8}$ & 74,4 & 25,6 & & & 5,0 \\
$\mathrm{M}_{15}$ & 1,3 & 22,7 & 47,8 & 23,2 & 4,6 \\
$\mathrm{E}_{15}$ & 0,5 & 12,0 & 50,1 & 32,8 & 6,3 \\
$\mathrm{C}_{15}$ & 1,7 & 21,0 & 44,7 & 26,3 & 10,4 \\
$\mathrm{SAC}_{15}$ & 51,3 & 48,7 & & & 11,5 \\
$\mathrm{M}_{22}$ & 0,6 & 12,4 & 43,2 & 33,4 & 13,5 \\
$\mathrm{E}_{22}$ & 0,4 & 6,5 & 37,6 & 44,0 & 35,8 \\
$\mathrm{C}_{22}$ & 0,4 & 11,3 & 39,0 & & \\
$\mathrm{SAC}_{22}$ & 39,6 & 60,4 & & & \\
\hline
\end{tabular}

${ }^{a}$ Escore ideal para sacro; ${ }^{b}$ escore ideal para musculosidade, estrutura física e conformação.

Para a consistência dos dados, o intervalo de idade dos animais seguiu uma variação de 150 a 330 dias em relação aos 240 dias (oito meses de idade), 360 a 540 dias em relação aos 450 dias (15 meses de idade) e 570 a 750 dias em relação aos 660 dias (22 meses de idade), considerando um período de 90 dias para menos e para mais em cada classe de idade. Foram eliminados os animais com medidas repetidas dentro de cada classe de idade. Para a verificação dos efeitos de ambiente que influenciam as características morfológicas, foram utilizados os procedimentos GLM e REG do software SAS/2004.
A definição dos grupos de contemporâneos (GC) para as características categóricas foi dada por: i) fazenda, ano e estação de nascimento, regime alimentar e lote de manejo aos 210 dias para os animais de oito meses de idade; e ii) fazenda, ano e estação de nascimento, lote de coleta para os animais de 15 e 22 meses de idade. Para as características contínuas de reprodução, os grupos contemporâneos contemplaram: (i) fazenda, ano e estação de nascimento, lote de manejo aos 210 e 365 dias para PE365; (ii) fazenda, ano e estação de nascimento, lote de manejo aos 210, 365 e 450 dias para PE450; e (iii) fazenda, ano e estação de nascimento para 
IPP. O efeito de estação de nascimento foi dividido em quatro classes: animais nascidos nos meses de janeiro a março, abril a junho, julho a setembro e outubro a dezembro.

A descrição dos arquivos de dados categóricos é apresentada na Tab. 2. Observa-se que há um maior número de dados para animais com idades mais avançadas, no entanto, isto se justifica pelo fato de que os animais avaliados visualmente por escores foram diferentes para cada faixa etária.
A estatística descritiva dos dados contínuos é apresentada na Tab. 3. É importante ressaltar que apenas $21 \%, 8 \%$ e $1 \%$ dos animais avaliados aos oito meses de idade também possuíam informações para o PE365, PE450 e IPP, respectivamente. Para os animais avaliados aos 15 meses de idade e com dados para PE365, PE450 e IPP, a porcentagem foi de 11\%, 16\% e $4 \%$, respectivamente. Aos 22 meses de idade, $8 \%, 13 \%$ e $10 \%$ dos animais apresentaram informações tanto para as características morfológicas como para PE365, PE450 e IPP.

Tabela 2. Descrição dos arquivos de dados analisados para as características musculosidade (M), estrutura física (E), conformação (C) e sacro (SAC) de bovinos da raça Nelore, para diferentes idades

\begin{tabular}{lcccc}
\hline \multirow{2}{*}{ Escore Visual } & \multicolumn{3}{c}{ Número } & GC \\
\cline { 2 - 4 } 8 & Animais & Touros & Vacas & \\
MEC de idade & 1.041 & 108 & 952 & 30 \\
SAC & 909 & 102 & 839 & 26 \\
& & & & \\
15 meses de idade & 1.894 & 216 & 1.749 & 75 \\
MEC & 1.666 & 191 & 1.566 & 67 \\
SAC & & & & \\
& & & 3.093 & 118 \\
meses de idade & 3.864 & 301 & 2.776 & 102 \\
MEC & 3.434 & 261 & & \\
SAC & & &
\end{tabular}

GC: grupo de contemporâneos.

Tabela 3. Estatística descritiva dos dados para as características contínuas perímetro escrotal (milímetros) aos 365 (PE365) e 450 (PE450) dias de idade e idade ao primeiro parto (meses, IPP) de bovinos da raça Nelore

\begin{tabular}{|c|c|c|c|c|c|c|}
\hline \multirow{2}{*}{ Variável } & \multicolumn{4}{|c|}{ Número } & \multirow{2}{*}{ Média $\pm \mathrm{DP}$} & \multirow{2}{*}{ CV (\%) } \\
\hline & Animais & Touros & Vacas & GC & & \\
\hline PE365 & 2.407 & 310 & 2.017 & 187 & $186,24 \pm 20,36$ & 10,94 \\
\hline PE450 & 2.401 & 330 & 2.401 & 211 & $211,03 \pm 24,97$ & 11,83 \\
\hline IPP & 8.238 & 533 & 5.642 & 227 & $38,70 \pm 4,87$ & 12,60 \\
\hline
\end{tabular}

GC: grupo contemporâneo; DP: desvio-padrão; CV: coeficiente de variação.

Os parâmetros genéticos das características categóricas morfológicas e características contínuas de reprodução foram estimados mediante análises bicaracterísticas sob modelo animal linear-limiar utilizando a estatística bayesiana por meio do aplicativo Multiple Trait Gibbs Sampler for Animal Models(MTGSAM) threshold, desenvolvido por Van Tassel et al. (1998). O modelo completo pode ser representado em notação matricial como: $\mathrm{y}=\mathrm{X} \beta+\mathrm{Z}_{1} \mathrm{a}+\mathrm{Z}_{2} \mathrm{c}+\mathrm{e}$, em que

$y$ é o vetor das observações (características contínuas e categóricas), $\beta$ é o vetor dos efeitos 
fixos (sexo e classe de idade da vaca ao parto para características morfológicas; grupo de contemporâneos e classe de idade da vaca ao parto para PE365 e PE450 ou somente grupo de contemporâneos para IPP), $a$ é o vetor dos efeitos aleatórios que representam os valores genéticos aditivos diretos de cada animal, $c$ é o vetor de efeitos aleatórios não correlacionados (efeito de grupos contemporâneos para as características morfológicas), $e$ o vetor de efeitos aleatórios residuais, e $X, Z_{1}$ e $Z_{2}$ são as matrizes de incidência que relacionam as observações aos efeitos fixos e aos efeitos aleatórios genético aditivo direto e não correlacionados, respectivamente. $\mathrm{O}$ arquivo de genealogia incluiu 52.361 animais da raça Nelore.

Os modelos de limiar geralmente apresentam problemas na estimação de componentes de variância e predição dos valores genéticos quando se tem um grande número de efeitos fixos (Varona et al., 1999; Luo et al., 2002). No entanto, estes problemas podem ser minimizados quando se considera o efeito de rebanho-anoestação como aleatório (Schaeffer et al., 2001). Porém, para que a convergência seja atingida, também é necessário que haja maior número de dados dentro de cada efeito fixo (Varona et al., 1999). Dessa maneira, o efeito de grupos de contemporâneos foi assumido como aleatório, e sexo e classe de idade da vaca ao parto como efeitos fixos para as análises genéticas de características categóricas.

No modelo de limiar assume-se que a escala subjacente apresenta distribuição normal contínua sendo representada como:

$\mathrm{U} \mid \theta \sim \mathrm{N}\left(\mathrm{W} \theta, \mathrm{I} \sigma_{\mathrm{e}}^{2}\right)$, em que

$U$ é o vetor da escala base de ordem $r ; \theta^{\prime}=\left(\beta^{\prime}\right.$, $\left.a^{\prime}, c^{\prime}\right)$ é o vetor dos parâmetros de locação de ordem $s \operatorname{com} \beta$ (definido sob o ponto de vista frequentista, como efeitos fixos) e ordem $s \operatorname{com} a$ e $c$ (como efeitos aleatórios genético aditivo direto e de grupo de contemporâneos); $W$ é a matriz de incidência conhecida de ordem $r$ por $s$; $I$ é a matriz de identidade de ordem $r$ por $r$; e $\sigma_{\mathrm{e}}^{2}$ é a variância residual. Dado que a variável na distribuição subjacente não é observável, a parametrização $\sigma_{\mathrm{e}}^{2}=1$ é, geralmente, adotada no sentido de se obter a identificabilidade na função de verossimilhança (Gianola e Sorensen, 2002). Tal pressuposição é padrão em análises desse tipo.

As características categóricas são determinadas por variáveis contínuas não observáveis, em escala subjacente, sendo fixados valores iniciais de limiares, em que $t_{1}<t_{2} \ldots<t_{j-1}$, com $t_{0}=-\infty$ e $t_{j}=\infty$, onde $j$ é o número de categorias. Os dados observáveis são dependentes da variável subjacente que é limitada entre dois limiares não observáveis (Gianola e Foulley, 1983). Assim, as categorias ou escores de $y_{i}$ (características morfológicas) para cada animal $i$ são definidos por $U_{i}$, na escala subjacente:

$$
y_{i}= \begin{cases}1 & t_{0}<U_{i} \leq t_{1} \\ 2 & t_{1}<U_{i} \leq t_{2} \\ 3 & t_{2}<U_{i} \leq t_{3} \\ 4 & t_{3}<U_{i} \leq t_{4} \\ 5 & t_{4}<U_{i} \leq t_{5} \quad i=1, \ldots, n\end{cases}
$$

em que $n$ é o número de observações. Após as especificações dos limiares $t_{0}$ a $t_{5}$, é necessário que um dos limiares (de $t_{1}$ a $t_{4}$ ) seja ajustado a uma constante arbitrária. Neste estudo, assumiuse $t_{1}=0$, tal que o vetor de limiares estimáveis foi definido como:

$$
t=\left[\begin{array}{l}
t_{2} \\
t_{3} \\
t_{4}
\end{array}\right]
$$

Para as análises bicaracterísticas em envolvem variáveis categóricas e contínuas, de acordo com o enfoque bayesiano, admitiu-se que as distribuições iniciais dos efeitos aleatórios genéticos, não correlacionados e residuais, seguem distribuição normal multivariada. Assumiram-se distribuições iniciais das (co)variâncias como uma Wishart invertida para os efeitos aleatórios genéticos, não correlacionados e residuais das características estudadas, inclusive a covariância entre elas. Foram definidas distribuições iniciais uniformes tanto para os efeitos fixos como para os limiares. $\mathrm{O}$ parâmetro $v$ é o grau de liberdade 
correspondente à distribuição Wishart invertida, indicando o grau de confiabilidade da distribuição inicial. Neste estudo, o parâmetro $v$ utilizado foi de valor zero, ou seja, não refletia nenhum grau de conhecimento sobre os parâmetros.

$\mathrm{Na}$ implementação da amostragem de Gibbs, foi utilizado um tamanho de cadeia inicial de 500.000 ciclos, sendo que os primeiros 100.000 ciclos foram descartados, e as amostras retiradas a cada 1.000 ciclos, totalizando 400 amostras iniciais. Em seguida, foi dada continuidade à análise, sendo adicionados 100.000 ciclos a cada rodada, até que as estimativas obtidas na última análise fossem iguais às obtidas na análise anterior. Esse critério foi utilizado com o intuito de verificar se realmente a convergência foi alcançada. As análises das amostras, da correlação serial e da convergência da cadeia de
Gibbs foram realizadas com o auxílio do programa GIBANAL (Van Kaam, 1998).

\section{RESULTADOS E DISCUSSÃO}

Para a obtenção das estimativas dos parâmetros genéticos, foi necessária uma cadeia de Gibbs de 600.000 ciclos para todas as análises bayesianas bicaracterísticas sob modelo animal linear-limiar. $\mathrm{Na}$ Tab. 4, são apresentadas as estatísticas descritivas das estimativas de correlação genética entre o perímetro escrotal padronizado aos 365 e 450 dias de idade e as características morfológicas de M, E e C, avaliadas aos oito, 15 e 22 meses de idade, em bovinos da raça Nelore. De modo geral, observa-se que as estimativas de correlações genéticas foram de sentido favorável à seleção, apresentando magnitudes moderadas, sugerindo que a seleção de indivíduos com melhor biótipo pode levar a animais com maior fertilidade.

Tabela 4. Estatística descritiva das estimativas de correlação genética para as características perímetro escrotal aos 365 (PE365) e 450 (PE450) dias de idade com musculosidade (M), estrutura física (E) e conformação (C), avaliadas aos oito, 15 e 22 meses de idade, em bovinos da raça Nelore

\begin{tabular}{|c|c|c|c|c|c|}
\hline Característica & Média & Moda & Mediana & $\mathrm{RC}(95 \%)$ & $\mathrm{EMC}^{\mathrm{DP}}$ \\
\hline \multicolumn{6}{|l|}{ PE365 } \\
\hline$+\mathrm{M}_{8}$ & 0,58 & 0,67 & 0,59 & 0,31 a 0,78 & 0,0055 \\
\hline$+\mathrm{E}_{8}$ & 0,47 & 0,47 & 0,47 & 0,17 a 0,72 & 0,0061 \\
\hline$+\mathrm{C}_{8}$ & 0,34 & 0,43 & 0,36 & $-0,08$ a 0,67 & 0,0086 \\
\hline$+\mathrm{M}_{15}$ & 0,55 & 0,62 & 0,57 & 0,28 a 0,75 & 0,0052 \\
\hline$+E_{15}$ & 0,17 & 0,18 & 0,18 & $-0,29$ a 0,61 & 0,0106 \\
\hline$+\mathrm{C}_{15}$ & 0,45 & 0,49 & 0,47 & $-0,02$ a 0,77 & 0,0091 \\
\hline$+\mathrm{M}_{22}$ & 0,46 & 0,50 & 0,48 & 0,12 a 0,70 & 0,0066 \\
\hline$+E_{22}$ & 0,50 & 0,44 & 0,45 & 0,16 a 0,68 & 0,0061 \\
\hline$+\mathrm{C}_{22}$ & 0,48 & 0,51 & 0,49 & 0,18 a 0,71 & 0,0057 \\
\hline \multicolumn{6}{|l|}{ PE450 } \\
\hline$+\mathrm{M}_{8}$ & 0,51 & 0,52 & 0,52 & 0,25 a 0,70 & 0,0051 \\
\hline$+E_{8}$ & 0,35 & 0,39 & 0,36 & $-0,02$ a 0,62 & 0,0066 \\
\hline$+\mathrm{C}_{8}$ & 0,24 & 0,32 & 0,26 & $-0,12$ a 0,57 & 0,0080 \\
\hline$+\mathrm{M}_{15}$ & 0,53 & 0,54 & 0,54 & 0,31 a 0,71 & 0,0045 \\
\hline$+E_{15}$ & 0,47 & 0,55 & 0,49 & 0,06 a 0,69 & 0,0070 \\
\hline$+\mathrm{C}_{15}$ & 0,56 & 0,61 & 0,59 & 0,23 a 0,78 & 0,0065 \\
\hline$+\mathrm{M}_{22}$ & 0,59 & 0,62 & 0,60 & 0,41 a 0,74 & 0,0042 \\
\hline$+E_{22}$ & 0,49 & 0,50 & 0,50 & 0,27 a 0,67 & 0,0045 \\
\hline$+\mathrm{C}_{22}$ & 0,65 & 0,66 & 0,65 & 0,50 a 0,77 & 0,0031 \\
\hline
\end{tabular}

RC: região de credibilidade a 95\%; EMC ${ }^{\mathrm{DP}}$ : desvio-padrão do erro de Monte Carlo.

O perímetro escrotal é um indicador de algumas características genéticas de interesse econômico, incluindo ganho em peso e fertilidade de machos e fêmeas (Paneto et al., 2002; Dias et al., 2006).
Neste contexto, os escores visuais apresentam inter-relações positivas e de grande magnitude com características de crescimento (Cardoso et al., 2001; Koury Filho, 2005; Kippert et al., 
2006), o que pode explicar as correlações genéticas favoráveis encontradas neste estudo para as características reprodutivas. Cyrillo et al. (2001), ao trabalharem com dados de bovinos da raça Nelore, estimaram as correlações genéticas entre medidas corporais e perímetro escrotal. Os autores concluíram que os genes que determinam a manifestação do perímetro escrotal são também, em parte, responsáveis pela manifestação de medidas corporais. Em trabalhos realizados buscando elucidar as relações entre os escores visuais e o crescimento, alguns autores encontraram estimativas de correlações genéticas moderadas a altas entre o peso $e$ as características de conformação, precocidade e musculosidade em bovinos da raça Nelore (Koury Filho, 2005) e Angus (Cardoso et al., 2004).

Na Tab. 4, observa-se também que as regiões de credibilidade para as estimativas de correlação genética de PE365 e PE450 com as características $\mathrm{C}_{8}, \mathrm{E}_{8}, \mathrm{C}_{15}$ e $\mathrm{E}_{15}$ apresentaram grande variação. A amplitude entre $\mathrm{o}$ valor máximo e mínimo do intervalo de confiança permite inferir, com maior confiabilidade, sobre as estimativas obtidas (Silva et al., 2003). Estes resultados podem indicar que os valores iniciais dos componentes de variância e as informações provenientes dos dados fenotípicos não foram suficientes. Em análises bayesianas, inferências são realizadas diretamente da distribuição posterior, baseando-se apenas nos dados disponíveis e na distribuição inicial. De acordo com Abdel Azim e Berger (1999), a acurácia das estimativas obtidas em modelos de limiar é maior quando se aumenta o número de categorias na característica, quando se tem uma distribuição mais próxima da normal ou quando houver número balanceado dos dados.

É importante ressaltar que apenas $21 \%$ e $8 \%$, $11 \%$ e $16 \%, 8 \%$ e $13 \%$ dos animais avaliados visualmente aos oito, 15 e 22 meses de idade também possuíam informações para o PE365 e PE450, respectivamente. No entanto, observa-se que o desvio padrão do erro de Monte Carlo foi muito pequeno para todas as análises, o que implica que o método bayesiano linear-limiar foi adequado na obtenção das estimativas de correlações genéticas em análises bayesianas bicaracterísticas. As estimativas de média, moda e mediana também foram semelhantes, exceto para algumas análises em que a estimativa da moda apresentou-se maior. Porém, é esperada uma distribuição Wishart Invertida das estimativas posteriores dos parâmetros genéticos, em análises multicaracterísticas (Sorensen et al., 1995), portanto, não é necessário que as medidas de tendência central sejam semelhantes.

As estimativas de correlação genética entre $o$ perímetro escrotal aos 450 dias de idade e as características morfológicas avaliadas aos 22 meses de idade foram maiores do que as obtidas entre as características morfológicas avaliadas aos oito e 15 meses de idade. Assim, a seleção para $\mathrm{M}_{22}, \mathrm{E}_{22}$ ou $\mathrm{C}_{22}$ permite obter animais mais eficientes em termos de fertilidade. A seleção aos 22 meses de idade pode ser tardia, porém, tais resultados refletem os sistemas de produção de bovinos de corte no Brasil que, em sua grande maioria, são de pastejo extensivo.

$\mathrm{Na}$ Tab. 5, são apresentadas as estatísticas descritivas das estimativas de correlações genéticas entre a idade ao primeiro parto e as características morfológicas de musculosidade, estrutura física, conformação e sacro, avaliadas aos oito, 15 e 22 meses de idade, em bovinos da raça Nelore. A correlação genética negativa, neste caso, implica associação favorável entre as características. Observa-se que as estimativas de correlações genéticas obtidas foram baixas, mas de sentido favorável, indicando que a seleção para animais com melhor biótipo pode levar à seleção de animais mais precoces. Vale ressaltar que a idade média ao primeiro parto na pecuária de corte no Brasil está acima de 40 meses de idade (Semmelmann et al., 2001). Assim, a identificação de fêmeas da raça Nelore que concebam em idades mais jovens é uma das prioridades de alguns programas de seleção e melhoramento da raça Nelore. De acordo com Patterson et al. (1992), os fatores ambientais, o peso vivo e a condição corporal são pontos importantes na redução da idade ao primeiro parto. Mercadante et al. (2000) relataram que a concepção é uma característica muito mais relacionada ao peso corporal do que à idade do animal, já que é prática comum, em muitos rebanhos, a adoção do peso mínimo para a entrada das fêmeas na reprodução. Tais afirmações sustentam os resultados obtidos neste estudo, uma vez que os escores visuais apresentam associação positiva com as características de crescimento. 
Tabela 5. Estatística descritiva das estimativas de correlação genética entre idade ao primeiro parto (IPP) com a musculosidade (M), estrutura física (E), conformação (C) e sacro (SAC), avaliadas aos oito, 15 e 22 meses de idade em bovinos da raça Nelore, obtidas a partir de análises bayesianas bicaracterísticas sob modelo animal linear-limiar

\begin{tabular}{lccccc}
\hline Característica & Média & Moda & Mediana & $\mathrm{RC}(95 \%)$ & EMC $^{\text {DP }}$ \\
\hline $\mathrm{M}_{8}$ & $-0,19$ & $-0,17$ & $-0,18$ & $-0,52 \mathrm{a} 0,11$ & 0,0076 \\
$\mathrm{E}_{8}$ & $-0,25$ & $-0,20$ & $-0,26$ & $-0,59$ a 0,13 & 0,0079 \\
$\mathrm{C}_{8}$ & $-0,37$ & $-0,34$ & $-0,38$ & $-0,63 \mathrm{a}-0,05$ & 0,0068 \\
$\mathrm{SAC}_{8}$ & $-0,23$ & $-0,27$ & $-0,25$ & $-0,64$ a 0,31 & 0,0115 \\
$\mathrm{M}_{15}$ & $-0,24$ & $-0,20$ & $-0,24$ & $-0,53$ a 0,08 & 0,0071 \\
$\mathrm{E}_{15}$ & $-0,17$ & $-0,30$ & $-0,19$ & $-0,60 \mathrm{a} 0,33$ & 0,0111 \\
$\mathrm{C}_{15}$ & $-0,35$ & $-0,35$ & $-0,37$ & $-0,66$ a 0,05 & 0,0087 \\
$\mathrm{SAC}_{15}$ & $-0,56$ & $-0,56$ & $-0,57$ & $-0,74$ a $-0,29$ & 0,0052 \\
$\mathrm{M}_{22}$ & $-0,46$ & $-0,49$ & $-0,47$ & $-0,66$ a $-0,20$ & 0,0052 \\
$\mathrm{E}_{22}$ & $-0,33$ & $-0,39$ & $-0,33$ & $-0,57$ a $-0,05$ & 0,0056 \\
$\mathrm{C}_{22}$ & $-0,49$ & $-0,51$ & $-0,50$ & $-0,68 \mathrm{a}-0,28$ & 0,0047 \\
$\mathrm{SAC}_{22}$ & $-0,50$ & $-0,50$ & $-0,51$ & $-0,69 \mathrm{a}-0,28$ & 0,0048 \\
\hline $\mathrm{RC}_{2}$ & & & & & \\
\hline
\end{tabular}

RC: região de credibilidade a 95\%; EMC $^{\mathrm{DP}}$ : desvio-padrão do erro de Monte Carlo.

As estimativas de correlação genética entre a idade ao primeiro parto e as características morfológicas $\mathrm{M}_{22}$, $\mathrm{E}_{22}$ e $\mathrm{C}_{22}$ apresentaram maior magnitude do que as obtidas para as características morfológicas aos oito e 15 meses de idade (Tab. 5), provavelmente porque são características com medidas mais próximas da idade ao primeiro parto. Além disso, os dados eram referentes às fêmeas criadas em regime de pastejo, o que pode ter favorecido idades mais altas ao primeiro parto, uma vez que essa característica é afetada, diretamente, pelo meio ambiente. No entanto, as estimativas de correlação genética foram semelhantes para $\mathrm{SAC}_{15}$ e $\mathrm{SAC}_{22}$, o que já era esperado, pois o sacro está sujeito a menor influência ambiental que os demais escores visuais avaliados neste estudo. Estes resultados confirmam os já relatados por Pita et al. (1998) e Semmelmann et al. (2001). Ao estudarem novilhas Nelore, Pita et al. (1998) observaram que as novilhas prenhes aos 18 meses tiveram maior frequência de escores visuais de três a cinco para conformação, precocidade e musculosidade em relação às novilhas não prenhes. Semmelmann et al. (2001), ao trabalharem com novilhas Nelore acasaladas aos 18 meses de idade, verificaram, ao sobreano, maiores escores visuais de conformação, precocidade e musculatura para as novilhas prenhes em relação às falhadas. Baseando-se em estimativas de correlação fenotípica, os autores concluíram que os escores visuais ao sobreano podem auxiliar na indicação da idade ao primeiro parto de fêmeas Nelore.

Conforme relatado por Mackinnon et al. (1990), as características de tamanho corporal e fertilidade têm suas expressões independentes, diferente dos resultados encontrados por Vargas et al. (1999), em trabalho realizado com animais da raça Nelore, em que as fêmeas foram classificadas em pequenas, médias e grandes, baseando-se na altura do posterior. Por essa classificação, os autores observaram diferenças significativas em desempenho reprodutivo entre os três grupos de diferentes tamanhos, com vantagens adaptativas, em ambiente à pasto, daquelas fêmeas de menor e médio tamanho.

De acordo com os resultados obtidos, as características morfológicas podem ser empregadas como critérios de seleção, com o intuito de aumentar a frequência de genes desejáveis para a fertilidade e precocidade. É importante ressaltar que não foram encontradas referências na literatura sobre estimativas de correlações genéticas entre características morfológicas, avaliadas por escores visuais, e as características reprodutivas. São necessários, portanto, mais estudos que permitam inferências mais seguras sobre estas estimativas. 


\section{CONCLUSÃO}

Os escores visuais para musculosidade, estrutura física e conformação devem ser utilizados como critérios de seleção com o intuito de obter progresso genético para fertilidade e precocidade sexual de bovinos da raça Nelore.

\section{REFERÊNCIAS BIBLIOGRÁFICAS}

ABDEL AZIM, G.A.; BERGER, P.J. Properties of threshold model predictions. J. Anim. Sci., v.77, p.582-590, 1999.

CARDOSO, F.F.; CARDELLINO, R.A.; CAMPOS, L.T. Fatores ambientais sobre escores de avaliação visual a desmama em bezerros Angus criados no Rio Grande do Sul. Rev. Bras. Zootec., v.30, p.318-325, 2001.

CARDOSO, F.F.; CARDELLINO, R.A.; CAMPOS, L.T. Componentes de (co)variâncias e parâmetros genéticos de caracteres pósdesmama em bovinos da raça Nelore. Rev. Bras. Zootec., v.33, p.313-319, 2004.

CYRILLO, J.N.S.G.; RAZOOK, A.G.; FIGUEIREDO, L.A. et al. Estimativas de tendências e parâmetros genéticos do peso padronizado aos 378 dias de idade, medidas corporais e perímetro escrotal de machos Nelore de Sertãozinho, SP. Rev. Bras. Zootec., v.30, p.56-65, 2001.

DIAS, J.C.; ANDRADE, V.J.; FRIDRICH, A.B. et al. Estimativas de parâmetros genéticos de características reprodutivas de touros Nelore, de dois e três anos de idade. Arq. Bras. Med. Vet. Zootec., v.58, p.388-393, 2006.

GIANOLA, D.; FOULLEY, J.L. Sire evaluation for ordered categorical data with a threshold model. Genet. Sel. Evol., v.15, p.201-224, 1983.

GIANOLA, D.; SORENSEN, D. Likelihood, bayesian, and MCMC methods in quantitative genetics. Statistics for Biology and Health. New York: Springer, 2002. 740p.

KIPPERT, C.J.; RORATO, P.R.N.; CAMPOS, L.T. et al. Efeito de fatores ambientais sobre escores de avaliação visual a desmama e estimativa de parâmetros genéticos, para bezerros da raça Charolês. Cienc. Rural, v.36, p.579-585, 2006.
KOURY FILHO, W. Escores visuais e suas relações com características de crescimento em bovinos de corte. 2005. 80f. Tese (Doutorado) Universidade Estadual Paulista, Jaboticabal, SP.

LÔBO, R.B. Programa de melhoramento genético da raça Nelore. Ribeirão Preto: FINEP, 1996. 100p.

LUO, M.F.; BOETTCHER, P.J.; SCHAEFFER, L.R. et al. Estimation of genetic parameters of calving ease in first and second parities of Canadian Holsteins using Bayesian methods. Livest. Prod. Sci., v.74, p.175-184, 2002.

MACKINNON, M.J.; HETZEL, D.J.S.; COBERT, N.J. Correlated responses to selection for cow fertility in a tropical beef herd. Anim. Prod., v.50, p.417-424, 1990.

MARCONDES, C.R.; PANETO, J.C.C.; SILVA, J.A.V. et al. Comparação entre análises para permanência no rebanho de vacas Nelore utilizando modelo linear e modelo de limiar. Arq. Bras. Med. Vet. Zootec., v.57, p.234-240, 2005.

MERCADANTE, M.E.Z.; LÔBO, R.B.; OLIVEIRA, H.N. Estimativas de (co)variâncias entre características de reprodução e de crescimento em fêmeas de um rebanho Nelore. Rev. Bras. Zootec., v.30, p.997-1004, 2000.

MEYER, K.; HAMMOND, K.; MACKINNON, M.J. Estimates of covariances between reproduction and growth in Australian beef cattle. J. Anim. Sci., v.69, p.3533-3543, 1991.

MWANSA, P.B.; CREWS, D.H.J.; WILTON, J.W. et al. Multiple trait selection for maternal productivity in beef cattle. J. Anim. Breed. Genet., v.119, p.391-399, 2002.

PANETO, J.C.C.; LEMOS, D.C.; BEZERRA, L.A.F. et al. Estudo de características quantitativas de crescimento dos 120 aos 550 dias de idade em gado Nelore. Rev. Bras. Zootec., v.31, p.668-674, 2002.

PATTERSON, D.J.; PERRY, R.C.; KIRACOFE, G.H. Management considerations in heifer development and puberty. J. Anim. Sci., v.70, p.4018-4035, 1992.

PEREIRA, E.; ELER, J.P.; FERRAZ, J.B.S. Análise genética de algumas características reprodutivas e suas relações com o desempenho ponderal na raça Nelore. Arq. Bras. Med. Vet. Zootec., v.53, p.720-727, 2001. 
PITA, F.V.; FRIES, L.A.; ALBUQUERQUE, L.G. Utilização de escores visuais como indicadores da idade à maturidade sexual de novilhas Nelore. In: SIMPÓSIO NACIONAL DE MELHORAMENTO ANIMAL, 2., 1998, Viçosa. Anais... Viçosa: SBMA, 1998. p.383384.

RAMIREZ VALVERDE, R.; MIZTAL， I.; BERTRAND, J.K. Comparison of threshold vs linear and animal vs sire models for predicting direct and maternal genetic effects on calving difficulty in beef cattle. J. Anim. Sci., v.79, p.333-338, 2001.

SCHAEFFER, L.; CALUS, M.; LIU, X. Genetic evaluation of conformation traits using random contemporary groups and reducing the influence of parent averages. Livest. Prod. Sci., v.69, p.129-137, 2001.

SEMMELMANN, C.E.N.; LOBATO, J.F.P.; ROCHA, M.G. Efeito de sistemas de alimentação no ganho de peso e desempenho reprodutivo de novilhas Nelore acasaladas aos 17/18 meses. Rev. Bras. Zootec., v.30, p.835$843,2001$.

SILVA, J.A.V.; ELER, J.P.; FERRAZ, J.B.S. et al. Análise genética da habilidade de permanência em fêmeas da raça Nelore. Rev. Bras. Zootec., v.32, p.598-604, 2003.
SORENSEN, D.A.; ANDERSEN, S.; GIANOLA, D. et al. Bayesian inference in threshold models using Gibbs sampling. Genet. Sel. Evol., v.27, p.229-249, 1995.

VAN TASSELL, C.P.; VAN VLECK, L.D.; GREGORY, K.E. Bayesian analysis of twinning and ovulation rates using a multiple - trait threshold model and Gibbs sampling. J. Anim. Sci., v.76, p.2048 -2061, 1998.

VAN KAAM, J.B.C.H.M. Gibanal 2.9: Analyzing Program for Markov Chain Monte Carlo Sequences. Wageningen: Dep. Anim. Sci., Wageningen Agricultural University, 1998.

VARGAS, C.A.; OLSON, T.A.; CHASE, C.C. et al. Influence of frame size and body condition score on performance of Brahman cattle. $J$. Anim. Sci., v.77, p.3140-3149, 1999.

VARONA, L.; MIZTAL, I.; BERTRAND, J.K. Threshold-linear versus linear-linear analysis of birth weight and calving ease using an animal model: I. Variance component estimation. $J$. Anim. Sci., v.77, p.1994-2002, 1999. 